\title{
HIGH-DISPERSION OPTICAL SPECTRA OF NEARBY STARS YOUNGER THAN THE SUN
}

\author{
Russel J. White, ${ }^{1}$ Jared M. Gabor, ${ }^{2}$ and Lynne A. Hillenbrand \\ Department of Astronomy, California Institute of Technology, Pasadena, CA 91125, USA; \\ russel.white@uah.edu, jgabor@orion.as.arizona.edu, lah@warning.caltech.edu \\ Received 2006 July 21; accepted 2007 January 15
}

\begin{abstract}
We present high-dispersion $(R \sim 16,000)$ optical $(3900-8700 \AA)$ spectra of 390 stars obtained with the Palomar 60 inch telescope. The majority of stars observed are part of the Spitzer Legacy Science Program "The Formation and Evolution of Planetary Systems." Through detailed analysis we determine stellar properties for this sample, including radial and rotational velocities, $\mathrm{Li}$ I $\lambda 6708$ and $\mathrm{H} \alpha$ equivalent widths, the chromospheric activity index $R_{\mathrm{HK}}^{\prime}$, and temperature- and gravity-sensitive line ratios. Several spectroscopic binaries are also identified. From our tabulations, we illustrate basic age- and rotation-related correlations among measured indices. One novel result is that $\mathrm{Ca}$ II chromospheric emission appears to saturate at $v \sin i$ values above $\sim 30 \mathrm{~km} \mathrm{~s}^{-1}$, similar to the well-established saturation of X-rays that originate in the spatially separate coronal region.
\end{abstract}

Key words: stars: activity - stars: fundamental parameters — stars: pre-main-sequence

Online material: machine-readable table

\section{INTRODUCTION}

We have obtained high-dispersion spectra of several hundred solar-type stars within $20-160 \mathrm{pc}$ of the Sun in order to determine fundamental stellar properties. Our work is in support of the Spitzer Legacy Program "Formation and Evolution of Planetary Systems" (FEPS; Meyer et al. 2006), which aims to understand the evolution of circumstellar dust from the primordial planetbuilding phase at an age of $\sim 3$ Myr to mature debris-disk systems up to an age of $\sim 3$ Gyr. While data from the Spitzer Space Telescope probe the dust characteristics, a wide range of ancillary ground-based observations are required to estimate stellar properties. High-dispersion spectroscopy, in particular, is a valuable tool for determining radial and rotational velocities, effective temperatures and surface gravities, abundances, and chromospheric activity diagnostics. These in turn provide useful constraints on stellar masses and ages. The stellar parameters derived from these spectroscopic observations thereby permit multiple axes of investigation for the infrared data from Spitzer, such as whether dust disk characteristics can be (anti)correlated with stellar mass, signatures of youth, and/or stellar multiplicity.

In $\S 2$ we describe the observed sample and the spectroscopic observations. In $\S \S 3$ and 4 we summarize the spectroscopic reductions and the methods used to extract spectroscopic properties. In $\S 5$ we discuss the spectroscopic binaries identified in the sample and illustrate basic age-related correlations among the measured properties; the relation and apparent saturation of chromospheric Ca II emission versus projected rotational velocity is highlighted. The analysis is meant to be representative rather than complete. The spectroscopic properties are presented primarily to assist more detailed studies of individual FEPS stars.

\section{SAMPLE AND OBSERVATIONS}

Our primary sample for high-dispersion spectroscopic observations consists of actual FEPS targets as well as many candidate targets eventually dropped from the FEPS program. The source list for FEPS is comprised of young near-solar analogs

\footnotetext{
${ }^{1}$ Currently at University of Alabama, Huntsville, AL 35899, USA.

2 Currently at University of Arizona, Tucson, AZ 85721, USA.
}

that range in mass from 0.7 to $1.5 M_{\odot}$ and span ages between 3 Myr and $3 \mathrm{Gyr}$. This source list was drawn from three samples of young solar analogs. The first sample was assembled from the D. R. Soderblom (2000, private communication) volumelimited ( $<50 \mathrm{pc})$ spectroscopic study of stars with $B-V$ colors between 0.52 and $0.81 \mathrm{mag}$ (spectral types $\sim \mathrm{F} 8-\mathrm{K} 0$ ) and $M_{V}$ magnitudes within 1.0 mag of the solar-metallicity zero-age main sequence; approximate ages are provided from the chromospheric

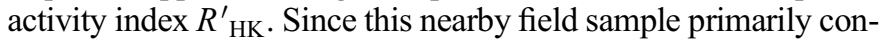
sists of stars older than a few $\times 10^{8} \mathrm{yr}$, a second sample was assembled with a targeted age range of 3-300 Myr. Stars in this sample were identified based on observational investigations to identify young main-sequence and pre-main-sequence solartype stars with strong X-ray emission, high lithium abundances, and kinematics appropriate for the young Galactic disk (e.g., Mamajek et al. 2002). The third sample consists of stars in nearby well-studied clusters, including four open clusters (IC 2602 [55 Myr], $\alpha$ Per [90 Myr], the Pleiades [125 Myr], and the Hyades [650 Myr]) and three younger $\mathrm{T}$ Tauri age clusters (R Corona Australis [3 Myr], Upper Scorpius [5 Myr], and Upper Centaurus Lupus [10 Myr]). Stars in these clusters serve to benchmark our field star selection criteria by providing subsamples nearly identical in age, composition, and birth environment. These three parent samples and the procedure for downselecting to the final FEPS sample observed with Spitzer are described more fully in Meyer et al. (2006). In addition to stars in this primary sample, a handful of other young and/or chromospherically active stars (e.g., RS CVn) were observed for comparison, and $\sim 20$ stars with precisely known radial velocities (Nidever et al. 2002) were observed for calibration and analysis purposes.

High-dispersion spectroscopic observations were obtained for 363 stars among the sample described above; 284 of these stars are among the 326 stars that comprise the final FEPS program as executed with Spitzer (the vast majority of FEPS stars not observed are in the southern hemisphere and thus inaccessible from Palomar Observatory). Fifty-two of the 363 stars observed are members of the four open clusters and 31 stars are members of the three young clusters. For calibration purposes, we also observed 13 giant stars with spectral types ranging from G5 to M2, five early- to mid-M dwarf stars, and nine early A-type stars with 
nearly featureless continua. Tables 1 and 2 list the observed sample (excluding nonsolar-type calibration stars), ordered by right ascension; Table 1 contains spectroscopic single stars and candidate single-lined spectroscopic binaries (for which stellar properties could be determined) while Table 2 contains known or newly discovered double-lined spectroscopic binaries. Coordinates for all stars are from the 2MASS All-Sky Catalog of Point Sources (Cutri et al. 2003), as interpreted by VizieR (Ochsenbein et al. 2000).

Spectroscopic observations were obtained on nine observing runs between 2001 July and 2003 June with the Palomar 60 inch $(1.5 \mathrm{~m})$ telescope and facility spectrograph (McCarthy 1988) in its echelle mode. This instrument is no longer available. A $1.43^{\prime \prime} \times$ $7.36^{\prime \prime}$ slit was used, yielding spectra spanning approximately $3900-8700 \AA$, with some small gaps between the redder orders. While the 2 pixel resolving power of this instrument is $\sim 19,000$, the achieved resolving power was typically $\sim 16,000$ because of image-quality issues. The wavelength coverage was chosen so as to include both the $\mathrm{Ca}$ II $\mathrm{H}$ and $\mathrm{K}$ features at the blue end and the $\mathrm{Ca}$ II infrared triplet at the red end. The spectrograph images the echelle spectra onto an $800 \times 800 \mathrm{CCD}$; the consequence of projecting the broad wavelength coverage onto this modest sized CCD is that the orders are closely spaced, especially at the red end. The detector has a gain of 1.5 and a read noise of $\sim 12$ electrons. During each night of observations, series of bias exposures and flat-field exposures were obtained to effect standard image processing. Because of the large spectral range of the instrument, the flat-field images were taken with two separate incandescent lamps, one appropriate for illumination of the blueward range of the detector (at $60 \mathrm{~s}$ exposure time) and the other appropriate for the redward range ( $5 \mathrm{~s}$ exposure time). For wavelength calibration, spectra of thorium-argon lamps were obtained (45 s exposure time) generally before or after each on-sky group of target exposures.

Each target observation consisted of between one and three consecutive exposures. The dates for these observations are listed in Tables 1 and 2; multiple-epoch observations are listed separately. At least three stars with precisely known radial velocities from Nidever et al. (2002) were observed per observing run.

One feature of this instrument is that the optimal focus for the bluest orders is not optimal for the reddest orders, so subjective compromises had to be made in focusing the spectrograph. In addition, the spectroscopic focus was temperature sensitive and therefore had to be tuned periodically throughout the night to achieve the best spectral resolution. On the night of 2002 February 2, many spectra were obtained at an exceptionally poor focus setting. When poorly focused, the spectrograph produces spectra that are stigmatic - spectral lines skewed relative to the dispersion direction, which degrades resolution (by an amount that is wavelength dependent). To help assess how poor focus may compromise the analysis (e.g., $v \sin i$ measurements), on the night of 2002 September 18 we observed the radial velocity standard HD 164992 over a broad range of focus values.

\section{SPECTROSCOPIC REDUCTIONS}

\subsection{Image Processing}

Raw data frames were processed using tasks in IRAF and custom IDL scripts. First, the detector bias was removed. Although the bias pattern appeared to be stable during the course of an observing run, the absolute bias level drifted substantially on timescales of hours, changing by as much as $30 \%$ over the course of a night. Bias subtraction was performed, therefore, in a two-step process. First, the bias sequence obtained on each night was median combined using the IRAF zerocombine task, rejecting the highest and lowest valued bias images. Second, since the detector bias level for a given image may have drifted from the median value determined at the beginning or end of the night, a bias scaling factor was also determined. This was done by measuring the median pixel value within a specified area in the most poorly illuminated, inefficient portion of the array, located well below $3600 \AA$ where useful spectra could not be extracted; even the brightest, bluest stars observed on our program showed essentially zero counts above bias in this portion of the array. For each target image, the median bias image from the beginning or end of the night was scaled by this factor and then subtracted.

Next, images were trimmed to exclude two unilluminated columns at the rightmost edge of the array. Cosmic-ray events were removed using IRAF's cosmicrays task and fairly liberal parameter settings that restricted the corrections to only bona fide cosmic rays.

The blue and red flat-field sequences from each night were median combined using IRAF's flatcombine procedure with $\sigma$-clipping. The blue and red medians were then stitched together approximately a third of the way up the detector (at line 250), which has the effect of distorting the one (curved) spectral order which intersects this line. IRAF's apflatten task was used to normalize the combined flat for each night by fitting its intensity along the dispersion using a low-order (typically 3 ) polynomial while setting all pixels outside an order to unity. Flat-fielding was performed by dividing each image by the normalized flatfield image using IRAF's ccdproc task.

Cosmetically, the images contain "tadpole streaks" of high count levels, comprised of short "heads" along the dispersion direction and then decaying count levels along long, mostly singlepixel "tails" perpendicular to the dispersion direction which thus affect multiple orders. The number of such defects per image was related to exposure time. A custom procedure was developed to crawl down each column comparing values on either side of the column and thus identifying these features. The pixels in the tail of the streak and a small square around the tadpole head were interpolated over.

The close spacing of the spectral orders did not permit measurement or removal of scattered light, an effect common in echelle spectrographs. However, comparisons of our extracted spectra with spectra previously obtained with other instruments $(\S 3.2)$ and of measured equivalent widths with those from the literature (§ 4.1) suggest that the majority of excess flux caused by scattered light is removed during background subtraction.

\subsection{Spectral Extraction and Wavelength Calibration}

One-dimensional spectra were extracted from the processed two-dimensional images using IRAF's doecslit task. Sixty orders were identified over the $800 \times 800$ pixel $^{2}$ array. A moderate (9th) order trace was employed and the spectra in each order were summed over 3-5 pixels perpendicular to the dispersion. Variance weighting was used for "optimal" extraction. The small separation between orders made removal of background and scattered light especially challenging. After exploring a variety of methods for accomplishing this, the most robust method identified was simply to subtract the average minimum value within two background regions, one on each side of the spectrum. Aperture definition, background region definition, and order tracing were attended as opposed to automated processes.

To test the accuracy of the adopted background/scattered-light subtraction, J. Valenti kindly provided a careful comparison of our spectra with spectra obtained with the HIRES spectrograph (Vogt et al. 1994) on the W. M. Keck I telescope. The widely spaced orders of HIRES permit accurate background and scattered-light 
TABLE 1

Spectroscopic Properties of Observed Single(-Lined) Stars

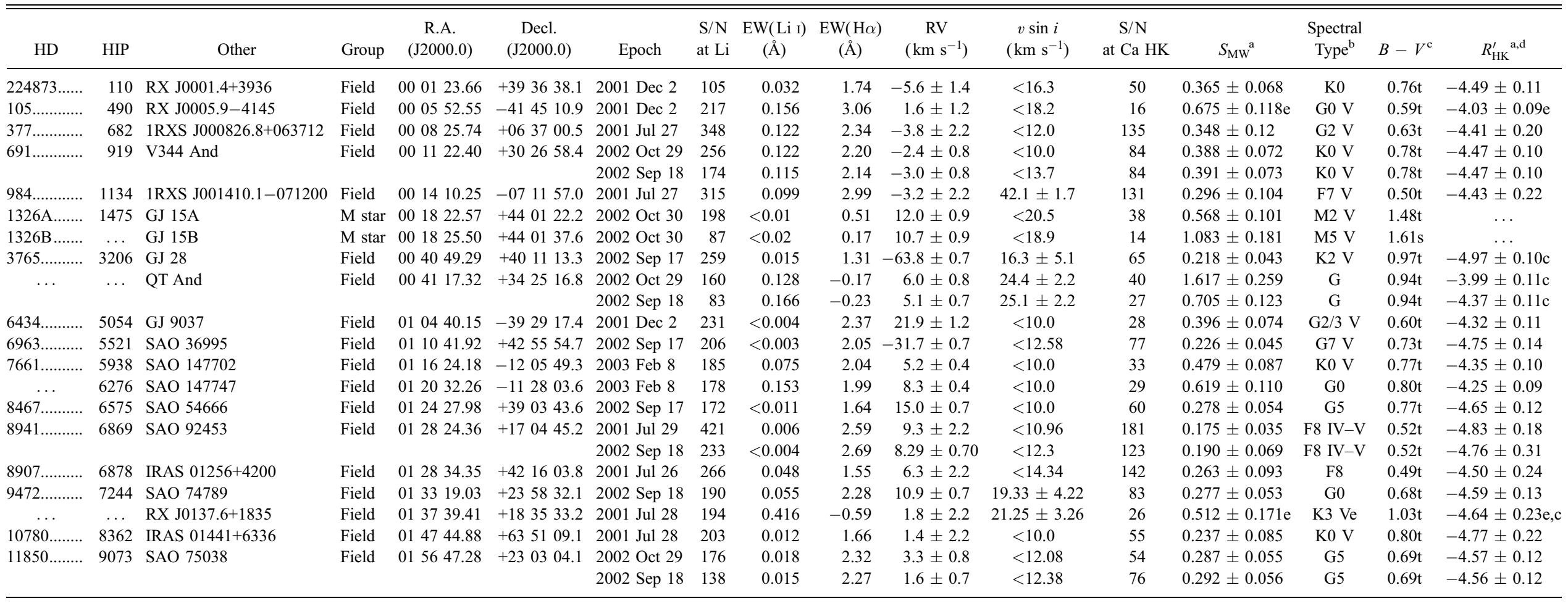

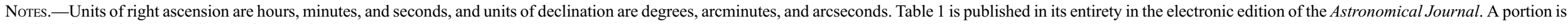
shown here for guidance regarding its form and content.

" Stars with especially uncertain $S$ values are marked with an "e".

b Stars which appear to be of low surface gravity are marked with an asterisk.

c $B-V$ colors from the Tycho catalog are marked with a " $\mathrm{t}$ " while those from the stellar spectral type are marked with an "s".

d Stars with $B-V$ colors outside the range over which $R_{\mathrm{HK}}^{\prime}$ values are calibrated are marked with a "c". 
TABLE 2

Observed Double-Lined Binary Stars

\begin{tabular}{|c|c|c|c|c|c|c|c|c|c|c|}
\hline HD & HIP & Other & Association & $\begin{array}{c}\text { R.A. } \\
(\mathrm{J} 2000.0)\end{array}$ & $\begin{array}{c}\text { Decl. } \\
(\mathrm{J} 2000.0)\end{array}$ & Epoch & $\mathrm{S} / \mathrm{N}$ at $\mathrm{Li}$ & $\begin{array}{c}\mathrm{EW}(\mathrm{Li} \mathrm{I}) \\
(\mathrm{m \AA} \AA\end{array}$ & $\begin{array}{c}\mathrm{EW}(\mathrm{Ca} \mathrm{I}) \\
(\mathrm{m} \AA)\end{array}$ & $\begin{array}{c}\mathrm{EW}(\mathrm{H} \alpha) \\
(\AA)\end{array}$ \\
\hline$\ldots$ & $\ldots$ & Cl Melotte 20848 & $\alpha$ Per & 032926.24 & +481211.74 & 2002 Oct 31 & 149 & $45 / 69$ & $61 / 85$ & $1.65 / 0.95$ \\
\hline $78899 \ldots \ldots \ldots \ldots$ & 45187 & GJ 3539 & Field & 091228.24 & +4912 23.4 & 2003 Feb 9 & 260 & $11 / 50$ & $91 / 121$ & $0.83 / 0.72$ \\
\hline \multirow[t]{3}{*}{$\ldots$} & \multirow[t]{3}{*}{$\ldots$} & \multirow[t]{3}{*}{ SAO 178272} & \multirow[t]{3}{*}{ Field } & \multirow[t]{3}{*}{095908.42} & \multirow[t]{3}{*}{-223934.57} & 2002 Feb 4 & 107 & $87 / 162$ & $105 / 153$ & Emission \\
\hline & & & & & & 2001 Dec 2 & 134 & $\ldots$ & $\ldots$ & Emission \\
\hline & & & & & & 2002 Apr 19 & 51 & $103 / 204$ & $174 / 233$ & $-0.1 /-0.2$ \\
\hline$\ldots$ & $\ldots$ & Tyc 73105031 & Field & 145837.69 & $\begin{array}{lll}-35 & 40 & 30.27\end{array}$ & 2003 Jun 5 & 82 & $121 / 222$ & $99 / 180$ & Emission \\
\hline \multirow[t]{2}{*}{$\cdots$} & \multirow[t]{2}{*}{$\cdots$} & \multirow[t]{2}{*}{ Tyc 73053801} & \multirow[t]{2}{*}{ Field } & \multirow[t]{2}{*}{145025.82} & \multirow[t]{2}{*}{-350648.66} & 2002 Feb 3 & 85 & $132 / 250$ & $103 / 66$ & $\ldots$ \\
\hline & & & & & & 2003 Jun 5 & 79 & $307 / 60$ & $120 / 54$ & $\ldots$ \\
\hline $140374 \ldots \ldots \ldots$. & 77081 & Tyc 733112351 & Field & 154421.06 & -331854.97 & 2003 Jun 4 & 145 & $112 / 116$ & $61 / 77$ & $-0.96 /-1.17$ \\
\hline $142229 \ldots \ldots \ldots$ & 77810 & SAO 121238 & Field & 155320.01 & +041511.5 & 2002 Feb 2 & 119 & $56 / 121$ & $29 / 79$ & $\ldots$ \\
\hline $218738 \ldots \ldots \ldots$ & 114379 & $\mathrm{KZ}$ And & Field & 230957.34 & +475730.0 & 2003 Jun 6 & 73 & $69 / 107$ & $108 / 165$ & $\ldots$ \\
\hline
\end{tabular}

NотE.-Units of right ascension are hours, minutes, and seconds, and units of declination are degrees, arcminutes, and arcseconds.

corrections. These comparisons demonstrated that the average scattered and/or background light remaining in our spectra is approximately $4 \%$ at $3900 \AA$, and drops roughly linearly to $0 \%$ at $6200 \AA$. The implication is that equivalent widths of features at blue wavelengths may be diminished by a small amount. Rather than attempt to correct for this in the reduction process, we account for this in the relative calibration of properties extracted from blue wavelength features ( $\S \S 4.3$ and 4.4 ).

Although multiple thorium-argon spectra per night were obtained (often one for each star observed), in practice we established wavelength calibration as follows. The extraction trace defined by a single bright star on each night was applied to its corresponding thorium-argon exposure to extract a one-dimensional wavelength reference spectrum. A wavelength solution was determined using the ecidentify task in IRAF, and this solution was applied to all spectra for a given night. This established wavelength solution was then interpolated onto a loglinear scale.

From analysis of spectra taken on the same night, small spectral shifts of $\sim 0.1 \AA$ \& were common, as inferred from comparisons of the location of telluric absorption lines. These shifts were likely due to flexure of the instrumental optics with telescope pointing, and consequently resulted in wavelength solution offsets of this order for many spectra. As discussed below, this wavelength offset is accounted for in determining radial velocities by cross-correlating telluric features.

\section{SPECTROSCOPIC PROPERTIES}

Figure 1 shows portions of the echelle spectra for seven stars near the $\mathrm{Ca}$ II $\mathrm{H}$ and $\mathrm{K}, \mathrm{Li}$ I 26708 , and $\mathrm{H} \alpha$ features. To help assess the quality of the spectra, two signal-to-noise ratios $(\mathrm{S} / \mathrm{Ns})$ are estimated, one at $\sim 6700 \AA$ and one at $\sim 3950 \AA$ (labeled as Li and HK, respectively, in Tables 1 and 2). The $\mathrm{S} / \mathrm{N}$ at $6700 \AA$ is determined from the gain-corrected median signal within the central portion of this order. The $\mathrm{S} / \mathrm{N}$ at $3950 \AA$ is determined similarly, but is based on the average of the values determined for the two adjacent orders containing the Ca II H (3968 $\AA$ ) and K (3934 $\AA$ ) features, and thus represents an approximate average over the continuum and broad absorption. These estimates are valid above values of $\gtrsim 20$, in which the noise is dominated by photon statistics. Lower values may actually overestimate the $\mathrm{S} / \mathrm{N}$, because of the increased relative error in the background, scattered-light, and bias-level subtractions $(\S 3.1)$. The $\mathrm{S} / \mathrm{N}$ distribution at $6700 \AA$ is shown in Figure 2.

The following sections described the procedures used to extract stellar properties from these spectra. In comparisons with previous measurements, values determined in this study are referred to as P60 values. Specifically, equivalent widths (EWs) of $\mathrm{Li}$ I $\lambda 6708$ and $\mathrm{H} \alpha$ are measured in $\S 4.1$, radial and rotational velocities are determined in $\S 4.2$, temperature and surface gravity indices are discussed in $\S 4.3$, and measured $S$ values and calculated $R_{\mathrm{HK}}^{\prime}$ indices are presented in $\S 4.4$.

\subsection{Equivalent Widths of $\mathrm{Li} \mathrm{I} \lambda 6708$ and $\mathrm{H} \alpha$}

Equivalent widths of the $\mathrm{Li}$ I $\lambda 6708$ doublet ( $\lambda 6707.76$, 26707.91), which is spectroscopically unresolved in our observations, and $\mathrm{H} \alpha \lambda 6562.8$ are measured for all spectra using the splot task within IRAF. For measurement of the Li I absorption feature which is located near the peak of the blaze function in these spectra, the local continuum is determined by fitting a Legendre polynomial to $\mathrm{a} \sim 30 \AA$ portion of the spectrum, excluding values less than $2 \sigma$ below the continuum (e.g., stellar absorption features) or more than $4 \sigma$ above the continuum (e.g., noise spikes) in this fit. The order of the polynomial is varied (typically from 4 to 7) to best match the local continuum. With the continuum defined in this way, the EW values are determined by fitting a Gaussian profile to the absorption feature. Multiple measurements on a single night are averaged, while separate epochs are listed independently. If no feature is discernible in the spectrum, upper limits are determined from the noise in the local continuum. Table 1 lists the measured values.

The measured $\mathrm{Li}$ I EW values include a contribution from an $\mathrm{Fe}_{\mathrm{I}}$ line at $6707.441 \AA$. Soderblom et al. (1993) find that the strength of this temperature-sensitive $\mathrm{Fe}$ I feature varies with $B-V$ color as $\mathrm{EW}\left(\mathrm{Fe}_{\mathrm{I}} \lambda 6707.441\right)=20(B-V)-3 \mathrm{~m} \AA$. Since the $B-V$ values of most stars are known or can be estimated ( $(4.4)$, the contribution of this $\mathrm{Fe}$ I line to the $\mathrm{Li}$ I EW values can be removed.

To assess possible systematic uncertainties caused by the subjective aspects involved in measuring $\mathrm{EW}$ values (e.g., continuum determination) or instrumental effects, we compare our measurements to previous values determined from high-dispersion spectroscopy. Of the spectroscopically single stars observed here, 21 Pleiads were observed by Soderblom et al. $(1993 ; R=50,000)$, 19 Hyads were observed by Thorburn et al. (1993; $R=26,000$ $32,000), 26$ field stars were observed by Wichmann et al. (2003; $R=40,000$ ), and 28 field stars were observed by Strassmeier et al. $(2000 ; R=25,000)$. The uncertainties in the $\mathrm{EW}(\mathrm{Li} \mathrm{I}) \mathrm{s}$ from these

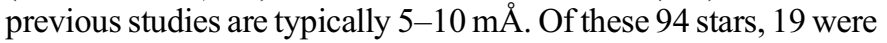
observed twice by us, yielding a total of 113 measurements for comparison. Figure 3 shows the difference between the P60 and 


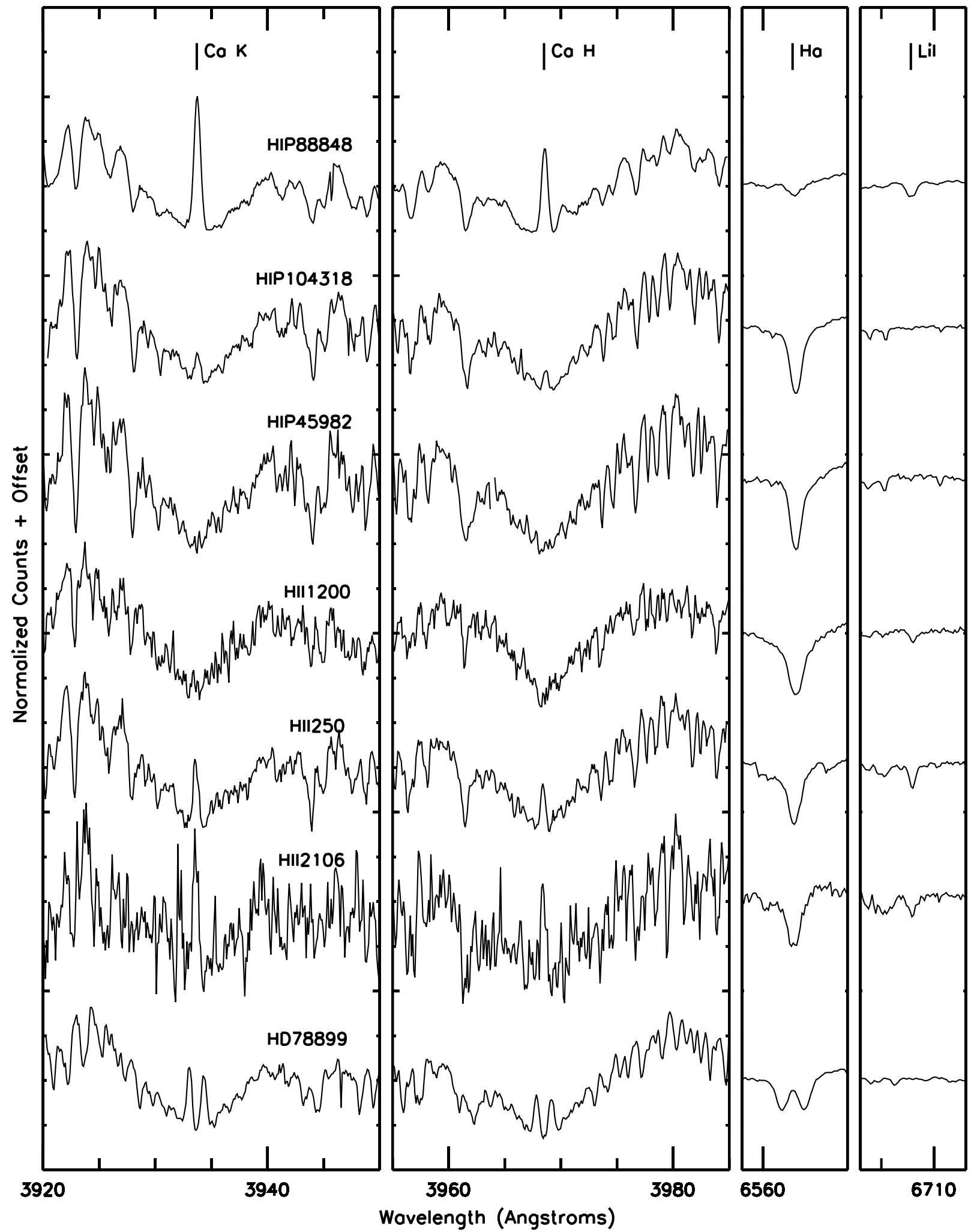

FIG. 1.-Portions of the extracted spectra. The top three spectra are G5 stars with decreasing levels of Ca II $\mathrm{H}$ and $\mathrm{K}$ emission $\left(\log R_{\mathrm{HK}}^{\prime}=-4.13,-4.59,-5.01\right)$. The next three spectra are Pleiads (and thus coeval), with spectral types of F5 (HII 1200), G2 (HII 250), and K0 (HII 2106). With a S/N of only 18 in the Ca II region, HII 2106 is typical of the poorest quality spectra from which $\log R_{\mathrm{HK}}^{\prime}$ values can be extracted; strong core emission can be seen in this case. The bottom spectrum shows the spectroscopic binary HD 78899, whose features are clearly doubled.

previous EW values; all values are as measured and thus uncorrected for the contamination of the $\mathrm{Fe}$ I line or potential scattered light. Overall the agreement is good. The average and/or median difference for any of the four studies is $\pm 15 \mathrm{~m} \AA$, with standard deviations of difference that range from 16 to $21 \mathrm{~m} \AA$. The average difference with the entire comparison sample is $-8 \mathrm{~m} \AA$ with a standard deviation of $20 \mathrm{~m} \AA$. We conclude that any systematic bias in our measurements is $\lesssim 10 \mathrm{~m} \AA$. We adopt a uniform uncertainty of $0.02 \AA$.

For $\mathrm{H} \alpha \mathrm{EWs}$, considerable care had to be taken both in normalizing to the local continuum and in fitting a functional form 


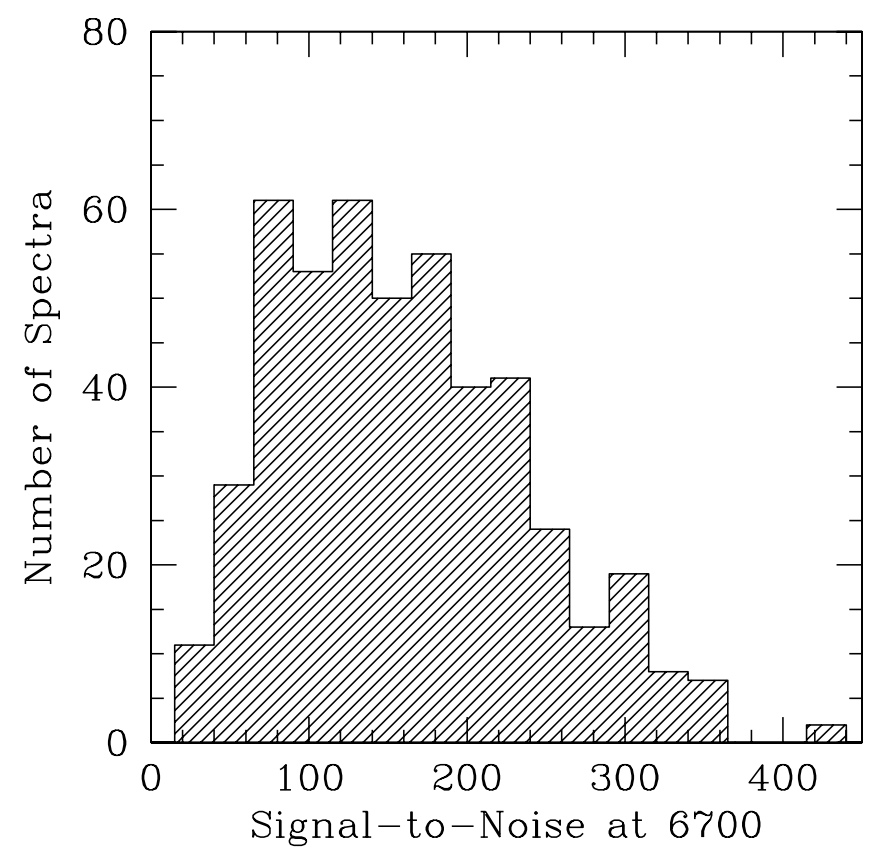

FIG. 2.- $\mathrm{S} / \mathrm{N}$ distribution of all spectra obtained (includes multiple measurements and stars observed for calibration) of the 390 unique targets.

to the line profiles because of the broad wings. The local continuum was determined by fitting a Legendre polynomial generally of order 10 to the entire spectral order, excluding values less than $2 \sigma$ below or more than $4 \sigma$ above the continuum. The procedure varied slightly as dictated by the noise level in the data, by the line breadth (because of, for example, rapid rotation), or if $\mathrm{H} \alpha$ was found to be in emission. Both the $\sigma$-rejection limits and the fitting order were modified in these cases to optimize the continuum fit. The EW values were determined by fitting a Voigt profile to the absorption feature; in a few cases of dramatically filled-in $\mathrm{H} \alpha$, direct integration was used to determine the $\mathrm{EW}$ rather than function fitting. EWs are listed in Table 1; negative values indicate $\mathrm{H} \alpha$ emission.

Four stars (HD 143006, RX J1842.9-3532, RX J1852.33700 , and LH98 196) show especially strong $\mathrm{H} \alpha$ emission (>10 A); their H $\alpha$ profiles are shown in Figure 4. In these cases, the strength and breadth of these emission-line profiles are greater than that expected from chromospheric activity alone, and more consistent with that expected from the accretion of circumstellar material (e.g., Muzerolle et al. 1998; White \& Basri 2003). We suggest that these stars are accreting.

For the eight stars identified as double-lined spectroscopic binaries, $\mathrm{EW}(\mathrm{Li}$ I $)$ s and $\mathrm{EW}(\mathrm{H} \alpha)$ s for the individual components were determined by fitting two Gaussian profiles to the doubled features, when possible. For these pairs, we also measured EWs of the temperature sensitive $\mathrm{Ca}$ I $\lambda 6717$ feature, which can be used to identify better the primary and the secondary. The EW values for the binaries are listed in Table 2 .

\subsection{Radial and Rotational Velocities}

Radial velocities and projected rotational velocities are determined via a cross-correlation analysis similar to that of White \& Hillenbrand (2004). The analysis is restricted to the 36 orders spanning $\sim 4000-7500 \AA$, excluding the 3 orders containing the telluric $\mathrm{B}$-band, $\mathrm{H} \alpha$, and $\mathrm{Na}$ II $\mathrm{D}$ features; orders at longer wavelengths have poorer wavelength solutions and those at shorter wavelengths typically have low $\mathrm{S} / \mathrm{Ns}$. From this restricted range, orders having an average $\mathrm{S} / \mathrm{N}$ greater than $\sim 25$ are then cross-

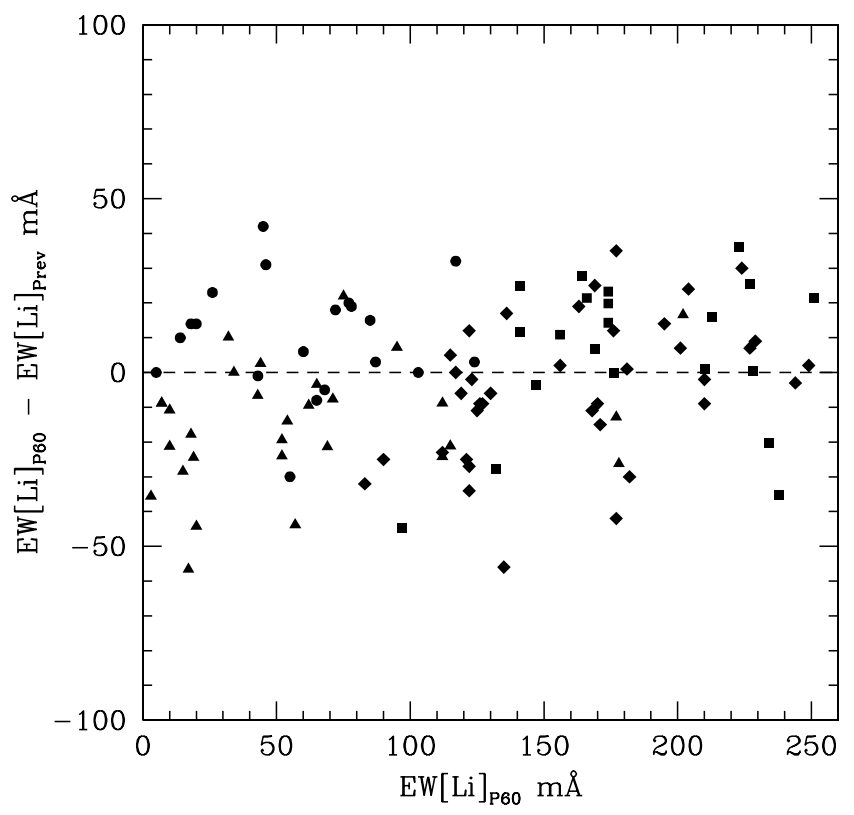

FIG. 3.-Comparison of measured EW( $\mathrm{Li}$ I 26708 ) values with values from Soderblom et al. (1993; squares), Thorburn et al. (1993; circles), Wichmann et al. (2003; diamonds), and Strassmeier et al. (2000; triangles).

correlated with the spectral orders of at least three slowly rotating comparison standards that have radial velocities accurate to $0.3-$ $0.4 \mathrm{~km} \mathrm{~s}^{-1}$ (Nidever et al. 2002). The radial velocity, relative to each standard, is then determined from the average velocity offset measured from all orders.

To determine the heliocentric radial velocities of the stars, the relative radial velocity must be corrected for barycentric motions and possible errors in the wavelength solution of either the standard or target spectrum. The former is determined using the rvcorrect task in IRAF. The latter correction is determined by cross-correlating the telluric $\mathrm{A}$ and $\mathrm{B}$ bands of the standard and the target star; the telluric correlations would yield an offset of zero for perfect wavelength calibration, but are typically $1-3 \mathrm{~km} \mathrm{~s}^{-1}$ for the spectra analyzed here. The multiple heliocentric radial velocity estimates, determined from multiple radial velocity standards, are then averaged to find the final radial velocity.

Uncertainties in the radial velocities are estimated from a combination of statistical and empirical error estimates. First, the uncertainty in the radial velocity relative to each standard is assumed to be the uncertainty in the mean value of all orders used, combined with the uncertainty in the radial velocity of the standard (typically $0.3 \mathrm{~km} \mathrm{~s}^{-1}$ ). The radial velocity estimates from all standards are then combined using a weighted average; the resulting statistical uncertainties are typically small $\left(0.1-0.3 \mathrm{~km} \mathrm{~s}^{-1}\right)$. As an empirical check on these uncertainty estimates, the radial velocity of each standard is determined from all other radial velocity standards observed during that run and compared to its assumed value. The average agreement of the radial velocity standards is typically a couple times larger than the statistical uncertainties, except in low$\mathrm{S} / \mathrm{N}$ cases where the statistical uncertainty dominates. This suggests there may be systematic effects unaccounted for in the statistical uncertainty estimates. Thus, we combine the statistical uncertainty with this empirical uncertainty to determine final (conservative) radial velocity uncertainty estimates given in Table 1. Figure 5 shows the distributions of radial velocities for the field, open cluster, and young cluster samples.

Rotational velocities $(v \sin i)$ for the observed targets are determined from the width of the peak in their cross-correlation with a 

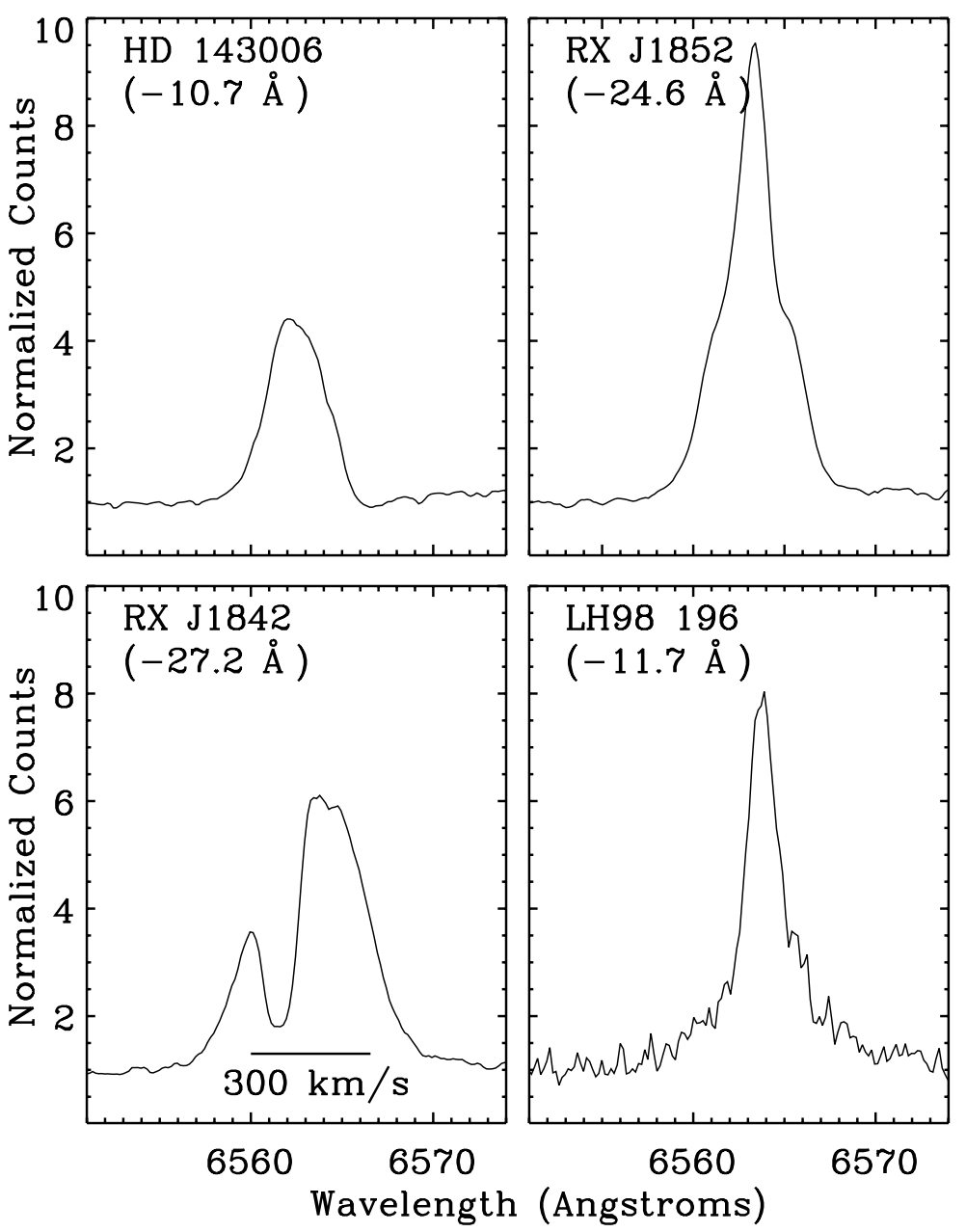

Fig. 4. - $\mathrm{H} \alpha$ profiles for the four strong emission-line stars normalized by the local continuum; the $\mathrm{H} \alpha$ equivalent width for each star is given in parentheses. A velocity width of $300 \mathrm{~km} \mathrm{~s}^{-1}$ is indicated.

slowly rotating standard. Specifically, the peak is fit by a parabola with the fitted width at half-peak maximum. To convert this width to a $v \sin i$ value, an empirical width versus $v \sin i$ relation is determined by cross-correlating many artificially "spun-up" spectra with the same slowly rotating standard. These artificially broadened spectra are constructed using the rotational profiles given in Gray $(1992 ; \epsilon=0.6)$. This procedure is computed for each order, yielding a $v \sin i$ estimate for each order. For each standard used, a $v \sin i$ value is computed from the median of all order estimates; a median is preferred since the $v \sin i$ measurements are often upper limits.

Uncertainties in $v \sin i$ are determined first from the standard deviation of the multiple $v \sin i$ measurements provided by all standards used. For observations consisting of multiple exposures, the uncertainty is the average standard deviation of these measurements. The $v \sin i$ values are assumed to be upper limits if less than $10 \mathrm{~km} \mathrm{~s}^{-1}$ (just over one-half the velocity resolution), or if $v \sin i$ is less than 3 times the uncertainty in $v \sin i$. We find that all templates of $\mathrm{F}, \mathrm{G}$, and $\mathrm{K}$ spectral types give consistent $v \sin i$ values; an exact match in spectral type is not critical in determining $v \sin i$.

As noted in the introduction, poor focus plagued some of our measurements, especially on the night of 2002 February 2. To assess how this may affect inferred properties, the above radial velocity and rotational velocity analysis was conducted on the observations of radial velocity standard HD 164922 observed at multiple focus settings $(\S 2)$. Fortunately the inferred radial velocities for modest focus offsets, typical of changes over a given night, agree with the nominal value to $\sim 1 \mathrm{~km} \mathrm{~s}^{-1}$, consistent with the inferred uncertainties. For extreme focus offsets, however, the discrepancy can be as large as $3 \mathrm{~km} \mathrm{~s}^{-1}$. Similarly, for modest focus offsets from nominal, the inferred $v \sin i$ values would increase modestly, but since the standard deviation of measurements among the orders correspondingly increased, the end result is simply larger upper limits (e.g., $<16 \mathrm{~km} \mathrm{~s}^{-1}$ for HD 164922 , compared with $<10$ when observed at nominal focus). However, for extreme focus offsets, the spectroscopic blurring resulted in a large apparent $v \sin i$ (e.g., $22.0 \pm 3.3 \mathrm{~km} \mathrm{~s}^{-1}$ for HD 164922). Since the majority of our spectra have at most only modest focus errors, no correction for this is made. Rotational velocities inferred from observations made on 2002 February 2, however, are marked with a colon in Table 1 as likely being artificially too large.

To empirically assess systematic errors in our radial and rotational velocity measurements, our values are compared with values measured by Nordstrom et al. (2004; which appeared after all of our data were taken). There are 135 stars in common between the surveys that (1) are not previously identified spectroscopic binaries, (2) are not used as radial velocity standards by us, and (3) have radial velocity uncertainties less than $4 \mathrm{~km} \mathrm{~s}^{-1}$. Figure 6 shows the difference between our radial velocities and those of Nordstrom et al. (2004). The agreement is quite good; the average difference is $-0.2 \mathrm{~km} \mathrm{~s}^{-1}$ with a standard deviation of $2.4 \mathrm{~km} \mathrm{~s}^{-1}$. With the exception of two stars, HIP 67904 and HIP 61072, all measured radial velocity values agree to within $10 \mathrm{~km} \mathrm{~s}^{-1}$. HIP 


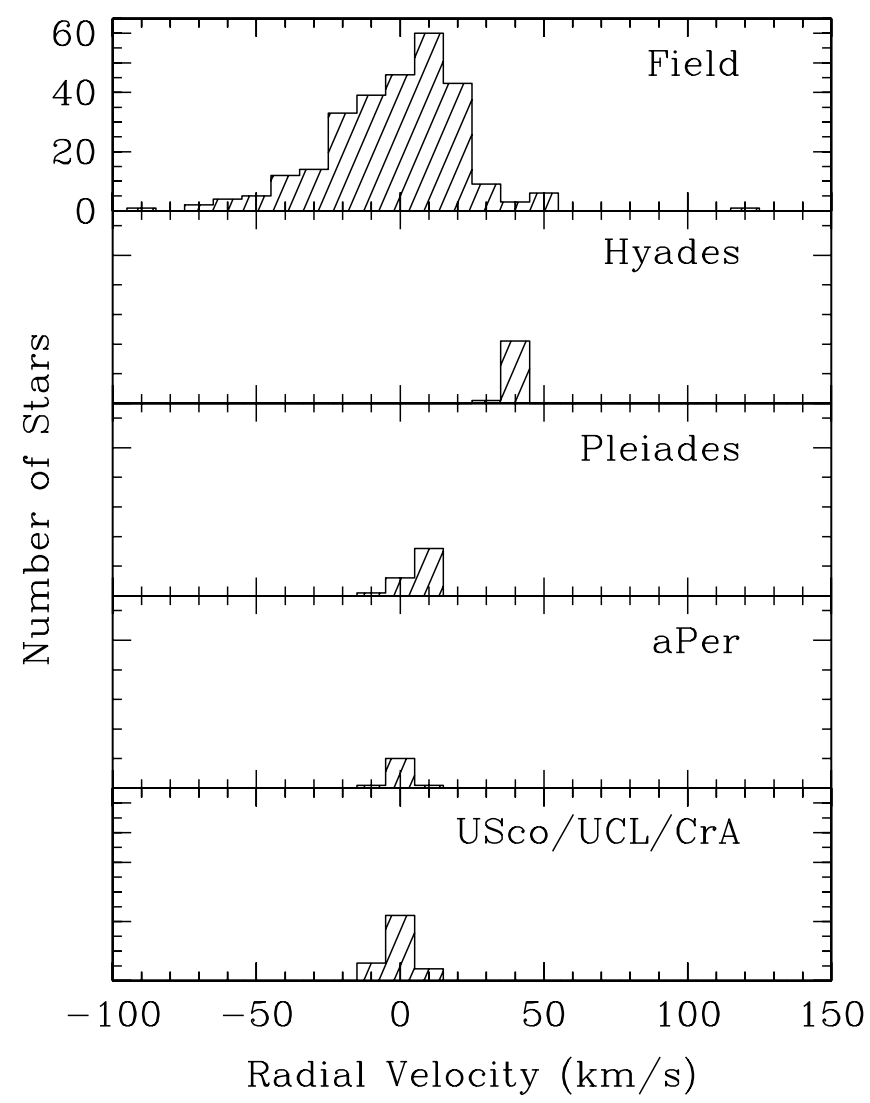

FIG. 5.-Distributions of radial velocities of the observed sample. The top panel shows the distribution for field stars and the bottom four panels show the distributions for cluster stars.

67904 and HIP 61072, on the other hand, have significantly different radial velocity measurements ( $>25 \sigma$ difference). As discussed below, we suggest these stars are spectroscopic binaries. This comparison also demonstrates that the radial velocities of stars with $v \sin i$ values as large as $45 \mathrm{~km} \mathrm{~s}^{-1}$ can be measured without significantly degraded precision.

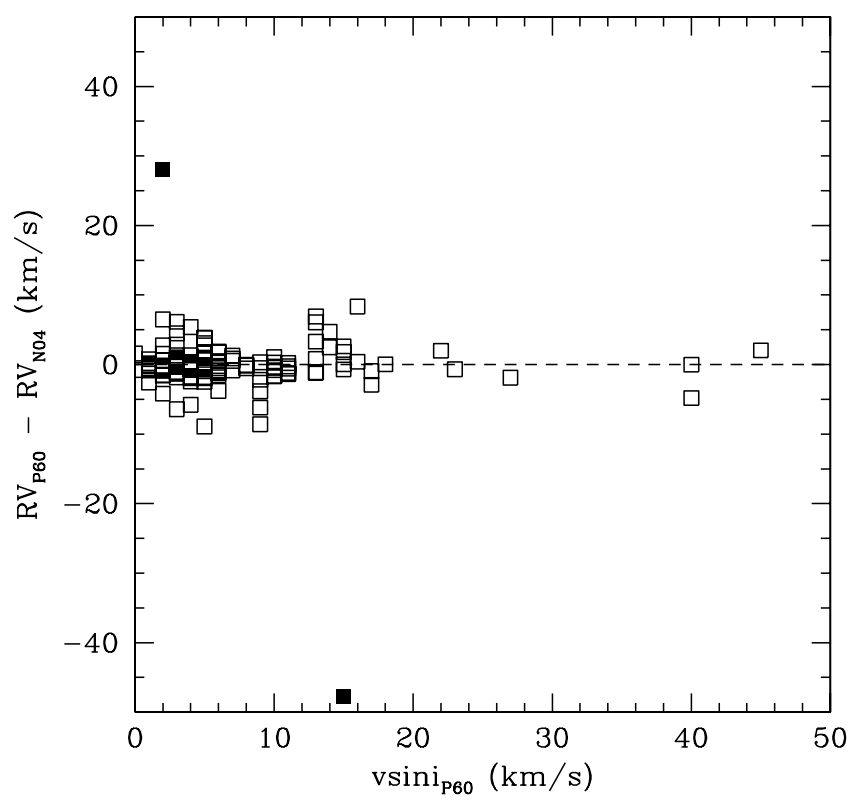

Fig. 6.-Difference between P60 radial velocities and values reported by Nordstrom et al. (2004). The filled squares are likely spectroscopic binaries.

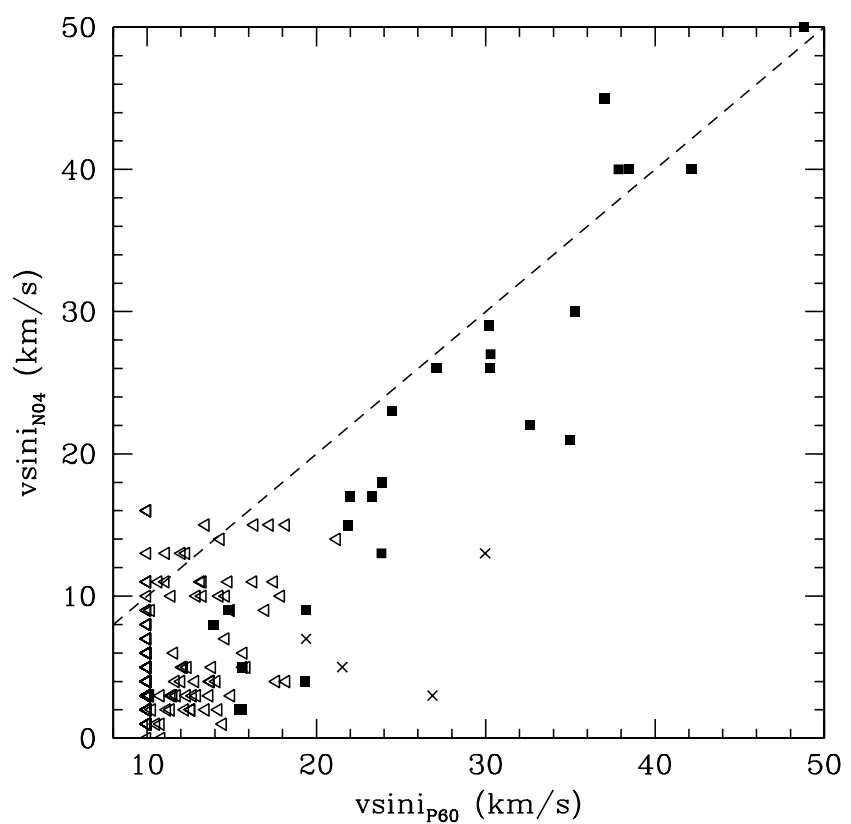

FIG. 7.-Comparison of measured $v \sin i$ values with values from Nordstrom et al. (2004). Triangles are upper limits and squares are measured values; Nordstrom et al. (2004) report no upper limits. The four crosses indicate measurements from the night of 2002 February 2, which had significant focus problems ( $§ 4.2)$.

In Figure 7 are shown Nordstrom et al. (2004) $v \sin i$ values versus the P60 $v$ sin $i$ values for the overlapping subsample described above. While Nordstrom et al. (2004) report $v \sin i$ values as low as $0 \mathrm{~km} \mathrm{~s}^{-1}$ (which is likely unrealistic), our lower resolution data restrict us to $v \sin i$ upper limits of $10 \mathrm{~km} \mathrm{~s}^{-1}$ or greater. The majority of our $v \sin i$ upper limits are thus greater than the values measured by Nordstrom et al. (2004), which inhibits interpretation of an overall comparison. However, a comparison of the 22 stars with measured values suggests a systematic difference. The P60 $v \sin i$ values are larger, in the median, than those of Nordstrom et al. (2004) by $8.6 \mathrm{~km} \mathrm{~s}^{-1}$. A portion of this difference may be a consequence of the spectroscopic blurring caused by the focus errors discussed above. When the three measured values from 2002 February 2 are removed, the P60 values are larger by only $6.3 \mathrm{~km} \mathrm{~s}^{-1}$ in the median. However, an independent comparison of the 389 stars with $v \sin i$ measurements in both Nordstrom et al. (2004) and Strassmeier et al. (2000), upper limits excluded, indicates that the Nordstrom et al. (2004) values are less than the Strassmeier et al. (2000) values by $2.6 \mathrm{~km} \mathrm{~s}^{-1}$. Thus, a portion of the difference with our measurements may be a consequence of artificially low values reported by Nordstrom et al. (2004). Overall we conclude that the majority of our measured $v \sin i$ values suffer from no systematic biases greater than $\sim 5 \mathrm{~km} \mathrm{~s}^{-1}$. The exceptions are stars observed on 2002 February 2, which may be biased toward artificially large values by $\sim 10-20 \mathrm{~km} \mathrm{~s}^{-1}$ because of especially poor focus. Since we are uncertain what the systematic correction to the $v \sin i$ values for these stars should be, if any, we make no correction for this in the values presented in Table 1.

\subsection{Temperature and Gravity Indicators}

Within the broad wavelength coverage provided by our echelle spectra are many temperature- and gravity-sensitive lines. Empirically, we find that the EWs of the photospheric features $\mathrm{Fe}$ I 24063, Fe I $\lambda 4071$, and Ca II $\lambda 4226$ (see Jaschek \& Jaschek $1987)$ are especially useful temperature diagnostics. In our data, 

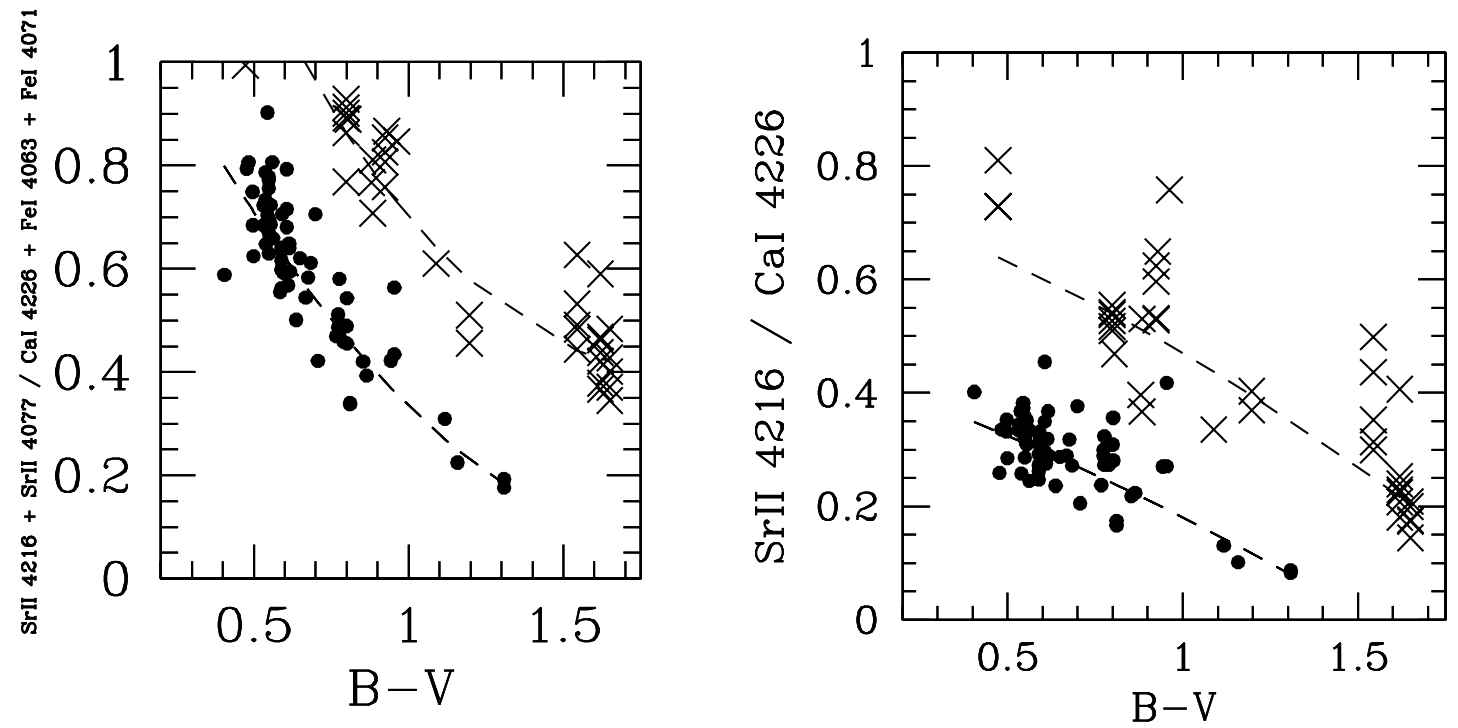

FIG. 8. - Gravity-sensitive equivalent-width ratios vs. $B-V$ color. Dwarf stars are indicated with filled circles and giant stars are indicated with crosses.

these features all increase in strength in a roughly linear fashion from spectral types F8 to K5, before degenerate flattening and turnover occurs toward later spectral types. However, fits of the EWs versus $B-V$ color for main-sequence stars (not shown here) are accurate to only $0.05-0.1$ in $B-V$. This implies that if we were to attempt spectral classification from the EWs, spectral-type uncertainties of more than one spectral subclass would result. Therefore we do not use these features to estimate spectral types. Spectral types instead are taken from several catalogs of stellar spectral classification, when available. These, in order of preference, are Houk \& Swift (1999), A. B. Skiff (2004), ${ }^{3}$ and Kharchenko (2001). The spectral types are listed in Table 1; 349 of the 370 stars observed have stellar spectral types.

For gravity indicators, we considered the ratios of the gravitysensitive Sr II $\lambda 4216$ and $\lambda 4077$ lines (see Jaschek \& Jaschek $1987)$ to the above temperature-sensitive lines. In Figure 8 we show for our dwarf and giant standard stars the ratios (Sr II $\lambda 4216+$ $\lambda 4077)$ to ( $\mathrm{Ca}$ I $\lambda 4226+\mathrm{Fe}_{\mathrm{I}} \lambda 4063+\mathrm{Fe}$ I $\left.\lambda 4071\right)$ and $\mathrm{Sr}$ II $\lambda 4216$ to $\mathrm{Ca}$ I $\lambda 4226$, as a function of $B-V$ color. These ratios are sensitive to both temperature and gravity; the dwarf and giant stars are reasonably well separated in these plots. Parabolic fits to the dwarf and giant points are shown. The majority of our target objects cluster around the dwarf locus, although some are securely between the dwarf and giant loci. Other diagnostic figures, such as $\mathrm{Sr}$ II $\lambda 4077 /\left(\mathrm{Fe}\right.$ I $\left.\lambda 4063+\mathrm{Fe}_{\text {I }} \lambda 4071\right)$ versus $\mathrm{Ca}$ I $\lambda 4226$ (which has the advantage of considering only our spectroscopic data), have similar appearances. In principle one might use such figures to quantitatively estimate the surface gravities of our program stars; in practice, however, the data do not allow this to be carried out with the precision normally expected of surface gravity measurements. Rather than estimate surface gravities, we simply identify all stars which are more than the $1 \sigma$ above the dwarf loci in both diagnostic planes. Sixty-six potentially low surface gravity stars are identified by these criteria and marked in Table 1.

\subsection{Chromospheric Ca II $H$ and $K$ Activity Measurements}

Core emission in the calcium $\mathrm{H}$ and $\mathrm{K}$ spectral lines serves as an indicator of stellar chromospheric activity, which is known to be correlated with stellar age (e.g., Soderblom et al. 1991), pri-

\footnotetext{
${ }^{3}$ Catalogue of Stellar Spectral Classifications (Vizier Online Data Catalog, III/233B).
}

marily as a consequence of rotation. We parameterize the core emission in our spectra using the standard Mount Wilson Project procedures (Wilson 1968; Vaughan et al. 1978). Our prescription for measuring the core emission closely follows that outlined by Duncan et al. (1991). First we define the index of core emission, $S$, as

$$
S=\frac{N_{H}+N_{K}}{N_{R}+N_{V}}
$$

where $N_{i}$ is the number of counts in band $i$. The continuum bands, $R$ and $V$, are $20 \AA$ wide and centered at 4001.07 and $3901.07 \AA$, respectively. The emission bands, $H$ and $K$, are centered on the emission features whose natural centers are at 3968.470 and $3933.664 \AA$, respectively. The bandpass for the $H$ and $K$ channels is triangular, with a full width at half-maximum of $1.09 \AA$. Before measuring the counts within these four bands, the spectra are normalized to account for the blaze function and shifted to correct for radial velocity. The spectra are normalized in a twostep iterative process. First, a $\sim 4$ th-order polynomial is fit to the spectra; the broad $H$ and $K$ absorption features are excluded in this fit. A second polynomial is then fit to the spectra, excluding all spectral features that extend $0.5 \sigma$ below the initial polynomial fit. The spectra are then normalized by dividing by this polynomial. The velocity shift is determined by cross-correlating the two spectra orders with the $\mathrm{Ca}$ II $\mathrm{H}$ and $\mathrm{K}$ features, with the spectrum of a bright comparison standard. The implied radial velocity is compared with that determined in $\S 4.2$ to ensure consistency; for the chromospheric activity analysis we adopted values determined directly from these orders as they typically provide the most accurate shift for these orders.

With velocity-shifted and normalized spectra, the counts within the continuum and emission bands of the $S$ index are summed. For the emission bands, the number of counts in each pixel is first multiplied by a number from 0 to 1 to mimic a triangular bandpass with a full width at half-maximum of $1.09 \AA$. With these sums, equation (1) is used to compute $S$.

To correct for systematic offsets in the measured $S$ values relative to previous work, possibly due to slight differences in the methodology or data quality, the $S$ values are compared to those measured by Wright et al. (2004; also appearing after all data for this program were gathered). These comparisons permit transformation 
of the $S$ values to what we refer to as Mount Wilson $S$ values, $S_{\mathrm{MW}}$. This is realized as a first-order regression analysis on $\log S$ in the form of

$$
\log S_{\mathrm{MW}}=A+B \log S \text {. }
$$

The uncertainty $\sigma_{S_{\mathrm{MW}}}$ is given by the standard formula

$$
\sigma_{S_{\mathrm{MW}}}^{2}=\left(\frac{\partial S_{\mathrm{MW}}}{\partial A} \sigma_{A}\right)^{2}+\left(\frac{\partial S_{\mathrm{MW}}}{\partial B} \sigma_{B}\right)^{2} .
$$

The analysis was performed on 8 of the 9 observing runs and for the entire overlapping sample; there were too few overlapping stars for the 2001 December observing run for comparison. Fortunately, the uncertainties in the implied correction estimates for 7 of the 8 observing runs agree well with that determined for the entire overlapping sample. The one exception was for the 2001 July observing run, for which the corrections appeared significantly different. Therefore, the corrections determined for this run were used to transform its $S$ values to $S_{\mathrm{MW}}$ values, while the remaining runs used the corrections determined for the entire sample, which are more precise. The resulting Mount Wilson calcium index values and uncertainties are listed in Table 1. Stars with uncertainties that are possibly much larger than reported, because of a large systematic uncertainty that is not formally propagated (e.g., radial velocity error), are marked with an " $\mathrm{e}$ " in Table 1.

Finally, the $S_{\mathrm{MW}}$ values are converted to $R_{\mathrm{HK}}^{\prime}$ values, which express the activity as a fractional ratio with the underlying star, following the prescription described in Noyes et al. (1984). However, this conversion requires proper characterization of the underlying photospheric flux, which can be assessed from the intrinsic stellar $B-V$ color. $B-V$ colors for most stars are obtained from the Tycho-2 catalog (Høg et al. 2000). These values are transformed to Johnson $B-V$ colors, following the relations in Mamajek et al. (2002). ${ }^{4}$ For objects without Tycho photometry, the $B-V$ colors are estimated from the stellar spectral type ( $\S 4.3)$ following the relations of Johnson (1966).

$R_{\mathrm{HK}}^{\prime}$ is calculated as the difference in the logarithms of the fluxcalibrated core emission $R_{\mathrm{HK}}$ and photospheric emission $R_{\text {phot }}$ :

$$
R_{\mathrm{HK}}^{\prime}=R_{\mathrm{HK}}-R_{\mathrm{phot}} .
$$

Here $R_{\mathrm{HK}}$ is calculated from the $S_{\mathrm{MW}}$ values and calibrated using the stellar $B-V$ color, and $R_{\text {phot }}$ is calculated from the $B-V$ color alone (see Appendix B in Noyes et al. 1984 for explicit formulae). Although the calibration of these terms is determined over the restricted $B-V$ range of $0.44<(B-V)<0.82$, we use it for the full $B-V$ range of the observed sample $[0.19<(B-$ $V)<1.65]$. The resulting $R_{\mathrm{HK}}^{\prime}$ values are listed in Table 1 .

\section{SAMPLE PROPERTIES}

\subsection{Spectroscopic Binaries}

Table 2 lists the eight stars with photospheric spectral features that are doubled in our observations. Two of these double-lined spectroscopic binaries were previously known (HE 848, SAO 178 272), while HD 78899, Tyc 7310503 1, Tyc 7305380 1, and HD 140374 are newly identified. The multiple-epoch observations of some stars also allow us to identify single-lined binaries based on changes in radial velocity. We consider an object a candidate binary if its radial velocity changed by more than $10 \sigma$ between observations; three stars meet this criteria: HD 92855

\footnotetext{
${ }^{4}$ There is a typographical error in the relations of Mamajek et al. (2002). The +0.007813 in eq. (C6) should be -0.007813 .
}
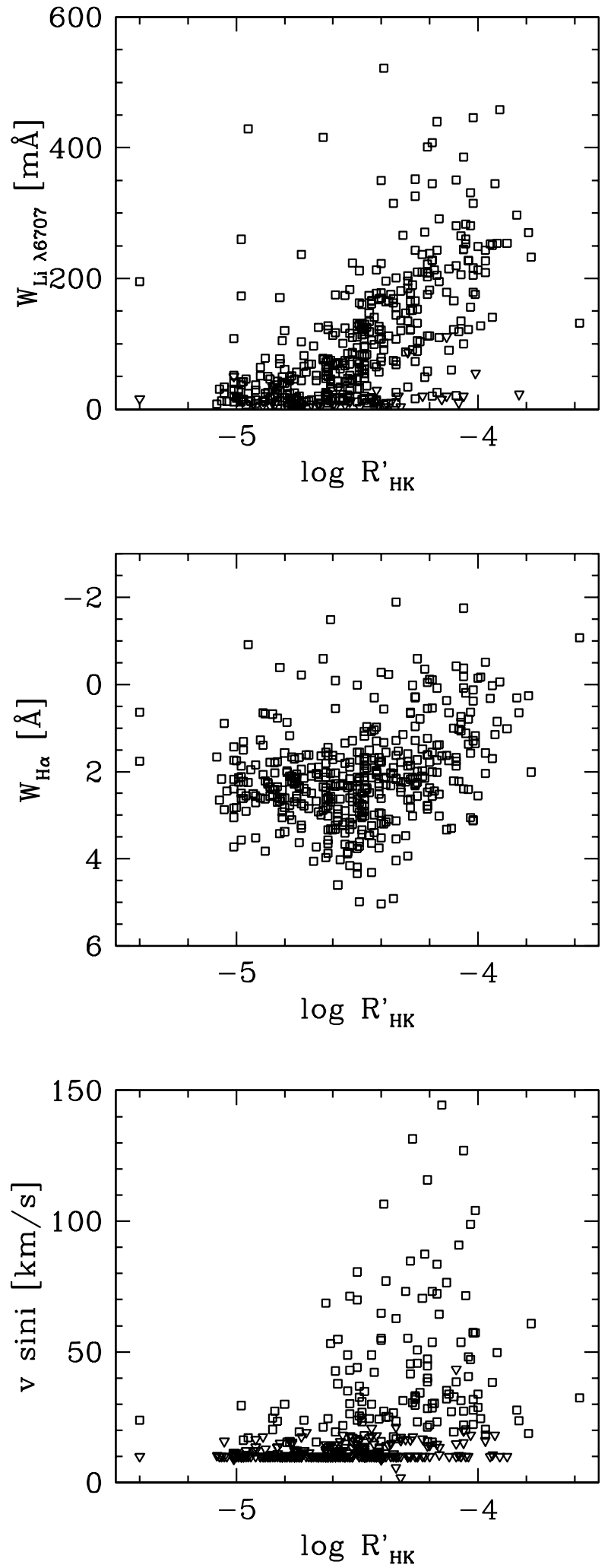

FIG. 9.- $\mathrm{EW}(\mathrm{Li} \mathrm{I}$ 26708), $\mathrm{EW}(\mathrm{H} \alpha)$, and $v \sin i$ vs. the chromospheric activity diagnostic $\log R_{\mathrm{HK}}^{\prime}$. Measured values are shown as squares while upper limits are shown as triangles.

(HIP 52498), HD 132173 (HIP 73269), and HD 61994 (HIP 38018; see Table 1). Similarly, based on comparisons with radial velocities published in Nordstrom et al. (2004) HD 108944 (HIP 61072) and HD 121320 (HIP 67904) are candidate binaries (see Fig. 6). All five are newly identified candidate binary systems. 

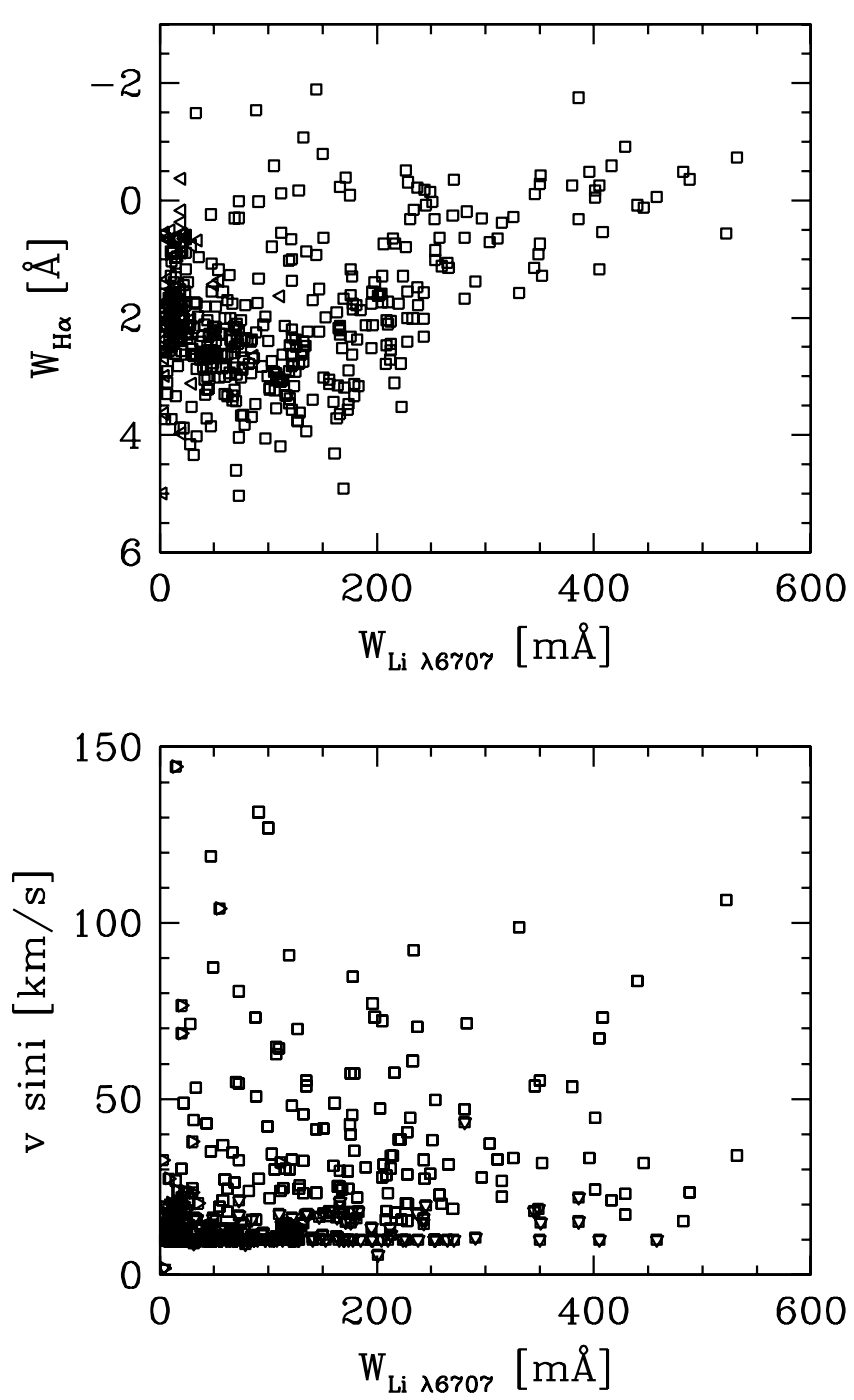

FIG. 10.- $\mathrm{EW}(\mathrm{H} \alpha)$ and $v \sin i$ vs. $\mathrm{EW}(\mathrm{Li}$ I 26708). Measured values are shown as squares while upper limits are shown as triangles.

Finally, since the radial velocity dispersion in young clusters is typically $<2 \mathrm{~km} \mathrm{~s}^{-1}$, comparison of cluster member radial velocity measurements with the median cluster values can identify single-lined spectroscopic binaries. Based on these comparisons, the two Pleiades members HII 571 and LH98 103 are identified as spectroscopic binaries; both have been identified previously as such (Mermilliod et al. 1992; Queloz et al. 1998).

\subsection{Sample Age Dispersion}

Several of the quantities extracted from the observed spectra are likely to be correlated with stellar age, based on extensive studies in the literature. Generally speaking, younger stars tend to be more rapidly rotating, have larger lithium abundances, and have stronger chromospheric emission lines such as $\mathrm{Ca}$ II $\mathrm{H}$ and $\mathrm{K}$, and $\mathrm{H} \alpha$. Thus the measured quantities $v \sin i, \mathrm{EW}(\mathrm{Li}$ I 26708$)$, $\log R_{\mathrm{HK}}^{\prime}$, and $\mathrm{EW}(\mathrm{H} \alpha)$ should correlate with age. Unfortunately the precision with which stellar ages can be estimated from these quantities is in general poor, in part because of the additional dependencies on stellar spectral type and rotation. Rather than attempting to disentangle these complicating effects for individual stars, we simply present the data to illustrate the empirical correlations among the measured quantities and to assess the overall age range of the observed sample.
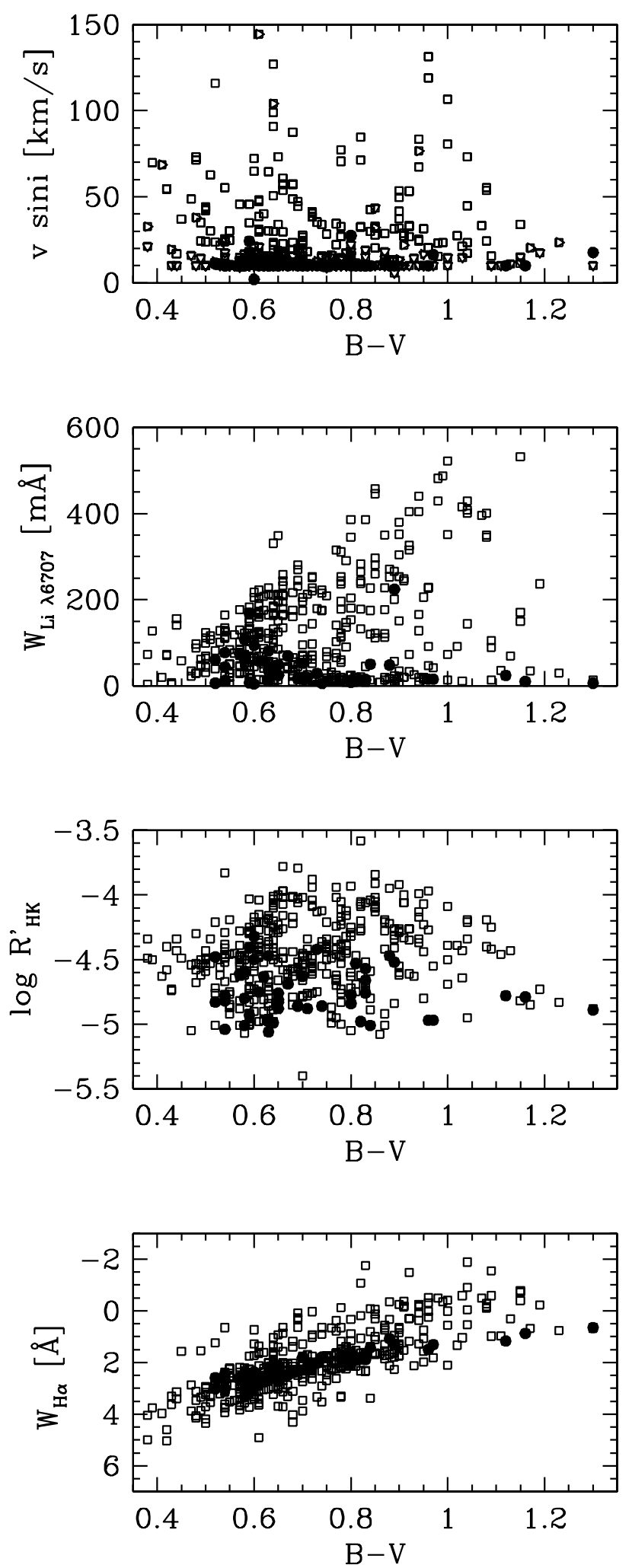

Fig. 11. - Projected rotational velocity $(v \sin i), \mathrm{EW}(\mathrm{Li} \mathrm{I} 26708), \log R_{\mathrm{HK}}^{\prime}$, and $\mathrm{EW}(\mathrm{H} \alpha)$ vs. $B-V$ color. Filled circles indicate dwarf standard stars. For clarity, a few stars in the observed sample are bluer than (SAO 41857) and redder than (HIP 108467, HIP 90190, and HIP 60783) the $B-V$ range shown here; the values are nevertheless included in Table 1. Similarly, in the bottom panel four stars with strong $\mathrm{H} \alpha$ emission $[\mathrm{EW}(\mathrm{H} \alpha)<-10$; Fig. 4] are not shown. 
Figure 9 shows the distributions of $\mathrm{EW}(\mathrm{Li}$ I 26708$), \mathrm{EW}(\mathrm{H} \alpha)$, and $v \sin i$ versus $\log R_{\mathrm{HK}}^{\prime}$. Error bars are not displayed due to crowding, but can be inferred from the information in Table 1. The EW( $\mathrm{Li}$ I) and $\log R_{\mathrm{HK}}^{\prime}$ values are reasonably well correlated, as expected if strong lithium absorption and strong chromospheric emission, as traced by $\mathrm{Ca}$ II emission, both indicate stellar youth. The $\mathrm{EW}(\mathrm{H} \alpha)$ and $\log R_{\mathrm{HK}}^{\prime}$ values are at best weakly correlated; stars with larger $\log R_{\mathrm{HK}}^{\prime}$ values have, on average, smaller $\mathrm{EW}(\mathrm{H} \alpha)$ values, suggesting they are partially filled in by emission. The strong spectral-type dependence of this temperature-sensitive line likely masks much of the underlying correlation which might be revealed by considering an analogously defined quantity $R_{\mathrm{H} \alpha}^{\prime}$. The $v \sin i$ values are well correlated, in the mean, with $\log R_{\mathrm{HK}}^{\prime}$ values. This is expected since increased rotation is believed to cause increased chromospheric activity, a characteristic common in young stars. Finally, in Figure 10 are shown the distributions of $\operatorname{EW}(\mathrm{H} \alpha)$ and $v \sin i$ versus $\mathrm{EW}(\mathrm{Li} \mathrm{I})$. Both the $\mathrm{EW}(\mathrm{H} \alpha)$ and $v \sin i$ values are correlated with $\mathrm{EW}(\mathrm{Li}$ I) in the mean, although both show a large scatter at all EW(Li I) values.

The above correlations corroborate the suggestion that these measured quantities trace stellar age. While the large scatter in these correlations inhibits assigning specific ages to individual stars, the ensemble distributions can help assess the age of the observed sample. Since the stellar temperature can also affect these quantities, we consider these distributions as a function of $B-V$ color, a proxy for stellar temperature. The range of observed values at any $B-V$ color should therefore (mostly) be a consequence of the age spread in the sample; a comparison of the spread of values at different colors can serve as a check for age biases with respect to temperature.

In Figure 11 are shown the distributions of $v \sin i, \mathrm{EW}(\mathrm{Li} \mathrm{I}$ 26708), $\log R_{\mathrm{HK}}^{\prime}$, and $\mathrm{EW}(\mathrm{H} \alpha)$ versus $B-V$ color (as assembled in $\S 4.4)$. The observed dwarf standards are distinguished in order to illustrate the properties of old slowly rotating stars. The distributions of $v \sin i$ and $\log R_{\mathrm{HK}}^{\prime}$ are independent of $B-V$ color, indicating a similar spread in rotation rate and chromospheric activity over the range of spectral types observed. The $\mathrm{EW}(\mathrm{Li}$ I) values span a broad range at each $B-V$ color. The larger $\mathrm{EW}(\mathrm{Li}$ I) values at the red end are a temperature effect; for a specified lithium abundance, the EW(Li I) increases with decreasing stellar temperature (Pavlenko \& Magazzú 1996). The $\mathrm{EW}(\mathrm{H} \alpha)$ values show the strongest $B-V$ trend, as expected for this temperature-sensitive line; the $\operatorname{EW}(\mathrm{H} \alpha)$ values decrease with increasing $B-V$ color. There is also a larger scatter at larger $B-V$ values. The scatter above the dwarf loci can be interpreted as stars with some $\mathrm{H} \alpha$ emission, whether this is partially or completely [e.g., $\mathrm{EW}(\mathrm{H} \alpha)<0.0]$ filling in the photospheric absorption line. Four stars with strong $\mathrm{H} \alpha$ emission [ $\mathrm{EW}(\mathrm{H} \alpha)>-10 \AA]$ are not plotted; their profiles are shown in Figure 4. Stars with some $\mathrm{H} \alpha$ emission span the full range of $B-V$ colors, although there appear to be many more at redder colors $(B-V>1.0)$ than bluer colors $(B-V<0.6)$. However, since an $\mathrm{EW}$ is defined relative to the continuum flux, a unique $\mathrm{EW}(\mathrm{H} \alpha)$ value corresponds to much less $\mathrm{H} \alpha$ flux for cooler (red) stars than hotter (blue) stars. Overall, these age indicators are consistent with the observed sample having ages that span from a few million years to a few billion years, as was intended. Moreover, all spectral types appear to span this range of ages; there is no strong age bias with spectral type among the observed sample.

\subsection{Ca II $H$ and $K$ Saturation in Young Stars}

While $\log R_{\mathrm{HK}}^{\prime}$ values have been shown to correlate with stellar age (e.g., Soderblom et al. 1991), this diagnostic typically has
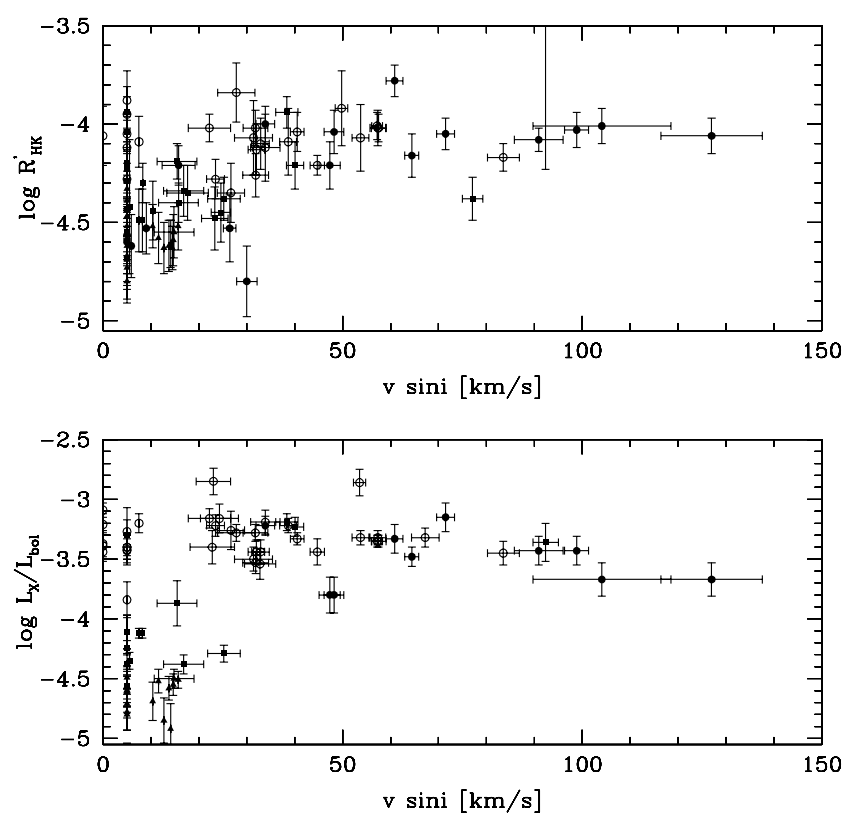

FIG. 12. $-R_{\mathrm{HK}}^{\prime}$ and $L_{\mathrm{X}} / L_{\text {bol }}$ measurements of open-cluster members vs. $v \sin i$. Filled circles are $\alpha$ Per members, squares are Pleiads, triangles are Hyads, and open circles are T Tauri stars in Upper Scorpius, Upper Centaurus Lupus, and R Corona Australis. Stars with a $v \sin i$ upper limit are plotted with a $v \sin i$ of $5 \mathrm{~km} \mathrm{~s}^{-1}$ for clarity.

not been used to estimate ages less than $\sim 100$ Myr. This primarily stems from the lack of $R_{\mathrm{HK}}^{\prime}$ measurements for stars in young clusters of known age, from which the age relation could be calibrated. Although the P60 $R_{\mathrm{HK}}^{\prime}$ measurements of young cluster members presented here may help establish this calibration, we illustrate that accurate ages will be inhibited by the broad range in rotation rates for stars at a given young age and the saturation of flux at high rotation rates.

In Figure 12 are shown the $R_{\mathrm{HK}}^{\prime}$ measurements versus projected rotational velocity $(v \sin i)$ of stars in the open clusters represented in our sample, including $\alpha$ Persei, the Pleiades, the Hyades, and collectively (post) T Tauri stars in Upper Scorpius, Upper Centaurus Lupus, and R Corona Australis. As expected, the $R_{\mathrm{HK}}^{\prime}$ measurements are correlated with $v \sin i$; the most rapidly rotating stars have, on average, the largest $R_{\mathrm{HK}}^{\prime}$ values. However, Figure 12 also indicates that once the $v \sin i$ values increase above $\sim 30 \mathrm{~km} \mathrm{~s}^{-1}$, the $R_{\mathrm{HK}}^{\prime}$ values stay roughly constant, implying no increase in the $\mathrm{Ca}$ II $\mathrm{H}$ and $\mathrm{K}$ emission-line flux toward larger rotation rates. A similar "saturation" phenomenon has been seen before based on observations of other emission lines originating in stellar chromospheres (O I $\lambda 1304, \mathrm{C}$ II 21335 ), as well as X-rays originating in stellar coronae (e.g., Vilhu 1984). It is not yet known whether the cause for flux saturation is internal (e.g., dynamo) or external (e.g., active regions) to the star (see Güdel 2004). Saturation of the $\mathrm{Ca}$ II $\mathrm{H}$ and $\mathrm{K}$ fluxes, as measured by the $R_{\mathrm{HK}}^{\prime}$ diagnostic, has not been demonstrated before.

While saturation of the $\mathrm{Ca}$ II $\mathrm{H}$ and $\mathrm{K}$ flux seems consistent with other chromospheric and coronal observations of rapidly rotating stars, we considered the possibility that this saturation is simply a consequence of the method used to determine the $S$ values, which are used to calculate $R_{\mathrm{HK}}^{\prime}$. Specifically, since the prescription outlined in $\S 4.4$ involves measuring the $\mathrm{Ca}$ II $\mathrm{K}$ and $\mathrm{H}$ emission with a triangular passband of specified width, apparent saturation could be caused by emission-line flux being rotationally broadened outside of this passband, and thus not included in the measurement. To test this, we remeasured $S$ values for a set 
of stars that were artificially rotationally broadened from 10 to $150 \mathrm{~km} \mathrm{~s}^{-1}$; we assume the core emission is rotationally broadened by the same amount as the photosphere. This showed that the technique used to measure the $S$ values begins to miss core emission flux above $v \sin i$ of $\sim 100 \mathrm{~km} \mathrm{~s}^{-1}$. Since the saturation observed for $R_{\mathrm{HK}}^{\prime}$ occurs well below this value, we interpret the saturation as a real effect.

To further demonstrate the case for saturation, in Figure 12 we also illustrate the ratio of X-ray flux to bolometric flux $\left(L_{\mathrm{X}} / L_{\mathrm{bol}}\right)$ for the stars in these clusters which have the available data. X-ray fluxes are from ROSAT observations and bolometric fluxes are determined from the atmospheric model which best fits the stellar energy distribution (see E. E. Mamajek et al. [2007, in preparation] for a complete description of the $L_{\mathrm{X}} / L_{\mathrm{bol}}$ derivation). A saturation trend is identified for coronal X-rays similar to that for chromospheric $\mathrm{Ca}$ II $\mathrm{H}$ and $\mathrm{K}$ core emission. The most rapidly rotating stars are the most fractionally X-ray bright, but $L_{\mathrm{X}} / L_{\mathrm{bol}}$ remains roughly constant above $v \sin i$ values of $\sim 30 \mathrm{~km} \mathrm{~s}^{-1}$.
This phenomenon has been demonstrated previously by many authors. What we have newly demonstrated here is that the same young cluster stars that have saturated $\mathrm{X}$-ray emission also have saturated chromospheric $\mathrm{Ca}$ II $\mathrm{H}$ and $\mathrm{K}$ emission.

We are grateful to the observing assistants formerly present at the Palomar 60 inch, Karl Dunscombe and Skip Staples, for their help and patience in acquiring this large data set. We thank E. Mamajek and C. Slesnick for assisting with the observations and Jonathan Foster, Francesca Colonnese, and Michael Inadomi for their assistance with data reduction and analysis. We appreciate greatly the scattered-light analysis provided by J. Valenti for some of our spectra. This research has made use of the SIMBAD and 2MASS databases. We acknowledge support for these observations from NASA contract 1224566 administered through JPL. This work has benefited from the ability to organize our data within the FEPS database created by John Carpenter.

\section{REFERENCES}

Cutri, R. M., et al. 2003, The IRSA 2MASS All-Sky Point Source Catalog (Pasadena: NASA/IPAC)

Duncan, D. K., et al. 1991, ApJS, 76, 383

Gray, D. 1992, The Observation and Analysis of Stellar Photospheres (Cambridge: Cambridge Univ. Press)

Güdel, M. 2004, A\&A Rev., 12, 71

Høg, E., et al. 2000, A\&A, 357, 367

Houk, N., \& Swift, C. 1999, Michigan Catalogue of Two-Dimensional Spectral Types for the HD Stars, Vol. 5 (Ann Arbor: Univ. Michigan)

Jaschek, C., \& Jaschek, M. 1987, The Classification of Stars (Cambridge: Cambridge Univ. Press)

Johnson, H. L. 1966, ARA\&A, 4, 193

Kharchenko, N. V. 2001, Kinematika Fiz. Nebesnykh Tel, 17, 409

Mamajek, E. E., Meyer, M. R., \& Liebert, J. 2002, AJ, 124, 1670

McCarthy, J. K. 1988, Ph.D. thesis, Caltech

Mermilliod, J.-C., Rosvick, J. M., Duquennoy, A., \& Mayor, M. 1992, A\&A, 265,513

Meyer, M. R., et al. 2006, PASP, 118, 1690

Muzerolle, J., Calvet, N., \& Hartmann, L. 1998, ApJ, 492, 743

Nidever, D. L., Marcy, G. W., Butler, R. P., Fischer, D. A., \& Vogt, S. S. 2002, ApJS, 141, 503

Nordstrom, B., et al. 2004, A\&A, 418, 989

Noyes, R. W., Hartmann, L. W., Baliunas, S. L., Duncan, D. K., \& Vaughan, A. H. 1984, ApJ, 279, 763

Ochsenbein, F., Bauer, P., \& Marcout, J. 2000, A\&AS, 143, 23

Pavlenko, Y. V., \& Magazzú, A. 1996, A\&A, 311, 961

Queloz, D., Allain, S., Mermilliod, J.-C, Bouvier, J., \& Mayor, M. 1998, A\&A, 335, 183

Soderblom, D. R., Duncan, D. K., \& Johnson, D. R. H. 1991, ApJ, 375, 722

Soderblom, D. R., Jones, B. F., Balachandran, S., Stauffer, J. R., Duncan, D. K., Fedele, S. B., \& Hudon, J. D. 1993, AJ, 106, 1059

Strassmeier, K., Washuettl, A., Granzer, Th., Scheck, M., \& Weber, M. 2000, A\&AS, 142, 275

Thorburn, J. A., Hobbs, L. M., Deliyannis, C. P., \& Pinsonneault, M. H. 1993, ApJ, 415, 150

Vaughan, A. H., Preston, G. W., \& Wilson, O. C. 1978, PASP, 90, 267

Vilhu, O. 1984, A\&A, 133, 117

Vogt, S. S., et al. 1994, Proc. SPIE, 2198, 362

White, R. J., \& Basri, G. 2003, ApJ, 582, 1109

White, R. J., \& Hillenbrand, L. A. 2004, ApJ, 616, 998

Wichmann, R., Schmitt, J. H. M. M., \& Hubrig, S. 2003, A\&A, 399, 983

Wilson, O. C. 1968, ApJ, 153, 221

Wright, J. T., Marcy, G. W., Butler, R. P., \& Vogt, S. S. 2004, ApJS, 152, 261 